\title{
Selection of Satellite Image Series for the Determination of Forest Pathology Dynamics Taking Into Account Cloud Coverage and Image Distortions Based on the Data Obtained from the Key Point Detector
}

\author{
E.O. Trubakov ${ }^{1}$, A.O. Trubakov ${ }^{1}$, D.A. Korostelyov ${ }^{1}$, D.V. Titarev ${ }^{1}$ \\ trubakoveo@gmail.com|trubakovao@gmail.com|nigm85@mail.ru|titaryovdv@mail.ru \\ ${ }^{1}$ Bryansk State Technical University, Bryansk, Russia
}

\begin{abstract}
Remote sensing of the earth and monitoring of various phenomena have been and still remain an important task for solving various problems. One of them is the forest pathology dynamics determining. Assuming its dependence on various factors forest pathology can be either short-term or long-term. Sometimes it is necessary to analyze satellite images within a period of several years in order to determine the dynamics of forest pathology. So it is connected with some special aspects and makes such analysis in manual mode impossible. At the same time automated methods face the problem of identifying a series of suitable images even though they are not covered by clouds, shadows, turbulence and other distortions. Classical methods of nebulosity determination based either on neural network or decision functions do not always give an acceptable result, because the cloud coverage by itself can be either of cirrus intortus type or insignificant within the image, but in case of cloudiness it can be the reason for wrong analysis of the area under examination. The article proposes a new approach for the analysis and selection of images based on key point detectors connected neither with cloudiness determination nor distorted area identification, but with the extraction of suitable images eliminating those that by their characteristics are unfit for forest pathology determination. Experiments have shown that the accuracy of this approach is higher than of currently used method in GIS, which is based on cloud detector.
\end{abstract}

Keywords: remote sensing of the earth, monitoring of forest pathology, image processing, descriptors of key points

\section{Introduction}

As provided for in the item 1 and 2, article 60.5 of the Forestry code of RF the state forest pathology monitoring is induced into the state ecological monitoring and represents a system of supervision by applying of both land, and remote methods in regard to sanitary state and forest pathologies (including testing, analysis and projected changes) [1].

The procedure for exercising the state forest pathology monitoring is enacted into law by the order of Ministry of Natura Resources of RF of 5 April 2017 N 156 «On approval of the Procedure for state forest pathology monitoring» [2]. According to this the task of remote observations concerning sanitary state and forest pathology is to identify changes in the sanitary state of forest and find forest pathologies alongside with preallotment and determination of the forest range boundaries and areas having these changes. Besides it, according to [2] it is determined that remote observation of sanitary state of the forest and forest pathology should be carried out through the interpretation of space images and aerial photographs obtained owing to the use of aircraft and unmanned aerial vehicles. This factor makes it necessary to obtain a set of images that can identify forest pathologies and monitor their changes.

Detection of forest pathologies by remote method is based on the fact that the stressed tree dries out in a vegetative way. Thus, we can say that the period of monitoring coincides with the period of vegetation in the area under observation. In the Central Federal District this period is approximately from 15 may to 15 October (about 5 months per year).

In this short period of time it is necessary to identify pathologies remotely and as quickly as possible, besides, it is important by using ground-based means to confirm them and to identify the causes. After that, if applicable, it's essential to get tough with the prevention of pathologies enlargement.

Vegetation dying off of the forest can occur due to various factors. Some of them can't be fought against (for example, cases of insufficient moisture). However, there are some other factors destroying forest, such as diseases (root rot). This disease follows long while lasting for years (about a decade). One more cause of forest dying is plant pests.

A striking example of needle-eating insects and leaf beetles is the Siberian silkworm (dendrolimus sibiricus), which is the most rampant in the forests of Siberia and the Far East. In these regions, millions of hectares of sound timber were lost because of the harmful activity of the insects. The time of gnawing of the tree crown spent by a silkworm depends on its population. For example, a population of one hundred species gnaw the needles of a tree within a period of about a day, if the population increases tenfold, the time is reduced to a few hours.

In Bryansk region, the main needle-eating pests or leaf beetles are sawflies and timber beetles. The population of the timber beetle on one tree can be up to several tens of thousands. With so many species it is able to destroy the tree within a month. That's why the European Union considers the beetle to be one of the most dangerous among secondary insects.

Thus, it can be concluded that, depending on the type of pest destroying trees, the development cycle of pathology varies from several years to several weeks. For this very reason a constant forest monitoring is indispensable.

Continuous monitoring of the forest implies examination of great number of satellite images taken for large areas. In this case taking into account the amount of data, manual image processing is neither suitable, nor effective, i.e. semi-automatic or automatic processing is required. However, the region of interest may be covered by clouds or other interference that make the dynamics of forest pathology erroneous.

Popular systems that provide unclosed series of satellite imagery [5], also contain information regarding areas covered by clouds (cloud mask). However, as shown, for example, in [4] the cloud mask Level 1C of Sentinel-2 images often omits the presence of clouds (the average error is $37.4 \%$ ). For the cases when satellite images contain either opaque clouds with a large transition zone (between the core of the cloud and the clean areas) or Cirrus clouds this error can be more than $50 \%$. The same situation takes place when using other systems that provide satellite imagery (Landsat, Wordview, etc.). Therefore, various approaches and algorithms in the field of cloud detection are being actively developed.

Most of modern methods of cloud zone detection in satellite images can be divided into three groups. The algorithms of the first group are based on calculations connected with certain circuit groups of multispectral images devoted to indices and characteristics and also decision functions applied to them $[9,10$, 14, 20]. They include the construction and analysis of histograms, threshold determination, as well as the analysis of deviations, etc. The second group includes algorithms of machine and deep machine learning based on artificial neural 
networks, classification trees, Kohonen maps, etc. [7, 8, 16, 17, $19,21]$.

Algorithms of these two groups give quite good results when detecting clouds in certain classes of satellite images, but often responds fallacious in more complex cases, when in satellite images there are different types of clouds and snow of complex geometric configuration. Therefore, in real-life situations, the algorithms of the third group, combining both approaches and detecting not only clouds, but also snow and shadow $[9,20]$ are applied. At the same time it is possible to achieve the recognition accuracy of about $90 \%$ [14].

However, it is worth noting that in addition to clouds there are other problems in satellite images that interfere with the successful detection of the forest pathology dynamics. They include defocusing, foreign objects and their shadows, turbulence, noise, etc. Consequently, such image may be unsuitable for analysis in spite of nil clouds conditions.

Thus, research in the field of methods and algorithms to automate the implementation of a satellite image series over a long period of time for forest pathology determination is required.

\section{Finding of forest pathologies in image series}

For automated determination of forest pathology dynamics it is necessary to have a sufficiently large image series concerning the region of interest. Images from the Copernicus open system are often used for this purpose [5]. From almost all satellite systems images of medium and high resolution are presented in the form of multispectral images. This feature of these images makes the selection of channels giving more information about typical objects under investigation possible, because it can exclude unnecessary information concerning foreign objects and emphasize the data which is important for the task.

Transformation of the image by the principal component method will allow allocating of the most significant reflected spectra, excluding chartjunks but without data loss. In this method, the first component emphasizes the spectral contrast as much as possible, while the second one reflects the boundaries of the contrast change. This approach allows improving the results of manual (visual) interpretation and automatic (semi-automatic) classification [3].

For the study of vegetation most indices are based on the difference between the spectral profile of healthy organics and the profile oppressed by diseases or pests. The most actively vegetating plant communities absorb more radiation in the red zone of the spectrum and reflect more in the near infrared scope compared to other objects [18]. The most commonly used standardized index NDVI [11]:

$$
N D V I=\frac{N I R-R E D}{N I R+R E D}
$$

where, $N I R$ is the reflection in the nearest infrared zone of the spectrum, $R E D$ is the reflection in the red region of the spectrum.

It should be borne in mind that the NDVI index is quite rough, especially with high and low canopy density. Therefore, it has been replaced by the less sensitive soil index MSAVI2 (Modified Soil Adjusted Vegetation Index 2) [12]:

$$
M S A V I 2=\frac{N I R-R E D}{N I R+R E D-L} *(1+L),
$$

where, $N I R$ is the reflection in the nearest infrared region of the spectrum; $R E D$ is the reflection in the red region of the spectrum, $L$ is the coefficient, which is by the formula:

$$
L=1-\frac{2 * N I R+1-\sqrt{(2 * N I R+1)^{2}-8 *(N I R * R E D)}}{2} .
$$

The official resource of the Copernicus project was used to obtain images in the conducted studies [5]. The selection of image series from this portal can be done interactively through search tools or using public API. In interactive mode, the search area of interest, dates of images, cloud parameters, types of satellites, types of images and so on, can be specified. The following parameters were used to study the proposed and standard methods:

1. The coordinates of the study area: (N53.44835, $\left.\mathrm{E} 34.26086^{\circ}\right)-\left(\mathrm{N} 53.40170^{\circ}, \mathrm{E} 35.37905^{\circ}\right)$.

2. Period: $2018-05-15$ - 2018-10-15 (the vegetation period in 2018).

3. Satellite Sentinel-2.

4. Cloudiness: $0 \%$.

With these search parameters 61 images are displayed in the results.

Similar results are obtained when using the OData API when prompted.

After analyzing the results visually, you can see that some images have noise (including elements of clouds). Due to such defects (noise, clouds), problems may arise in the following analysis of the images. If images with defects are immediately used for forest pathologies recognizing, the probability of erroneous results increases for both: monitoring and training tasks. Therefore, it is necessary to carry out additional processing with a series of received images to filter out noisy and defective ones. Often 1-2 images with noise or clouds in the analyzed area can lead to an erroneous forecast of forest pathology dynamics.

\section{Detection of images suitable for determining the forest pathology dynamics}

Experiments have proved that the currently used approaches to determine images without clouds and distortion, used in Copernicus or in a number of modern GIS (eg QGIS) make a number of errors. For example, for the conditions described in the previous paragraph, the Semi-Automatic Classification Plugin (SAC) from the QGIS package ( that is used very often to solve similar problems) has identified 6 "ideal" images over the past 5 months (with the criterion of nil clouds). Besides, the frequency of images is irregular (having intervals in images of 54 days). These indicators in some types of pathologies are insufficient.

It is worth noting that among the images removed by the module there are those that could be used to analyze the dynamics of forest pathology. Most often these are the images which are partially covered by clouds, but the area of interest in them is not distorted. It should be mentioned that the manual selection of a series of images in practice is time-consuming and is not popular.

The paper proposes an alternative approach to the selection of a series of images. The main idea of the approach is that it involves proceeding not from the parameters of clouds or distortions, but from the parameters of the area of interest and its specific points and parameters. To do this, it is proposed to use key points based on one of the known descriptors. In this case, the image is considered suitable for a series of images if and only if a predetermined percentage of key points of interest, calculated from the reference image can be found in the candidate image.

The proposed algorithm consists of two stages.

Stage 1-pre-training in the initial image:

1. Manual selection of the single reference image that shows the area of interest.

2. Marking of the area of interest. It is important to take into account that the area should not be too small (otherwise the number of key points will not be sufficient) for the stable operation of the algorithm.

3. Obtaining reflection layers in the near infrared and red spectral region $(N I R, R E D):\{\mathrm{B} 04, \mathrm{~B} 8 \mathrm{~A}\}$.

4. Formation of a monochrome image by the algorithm MSAVI2:

$$
M S A V I 2=\frac{N I R-R E D}{N I R+R E D-L} *(1+L) .
$$


5. Calculation of key points of interest, computation of their descriptors.

After the first stage we get a reference vector of descriptors regarding key points of interest

Stage 2- automated selection of a series of images with neither distortions nor clouds covering of the area of interest:

1. Getting a snapshot from Copernicus system via internal API.

2. Selection of reflection layers from the obtained image in the nearest infrared and red spectral regions (NIR, RED).

3. Selecting an area by applying a mask to the image using the logical «AND » operation by means of a single mask of the area of interest: Res $=I m g$ \& Mask.

4. Getting of layers in the spectra $R E D, N I R:\{\mathrm{B} 04, \mathrm{~B} 8 \mathrm{~A}\}$

5. Formation of a monochrome image according to the transformation algorithm MSAVI2.

6. Calculation of key points and their descriptors.

7. Comparison of the obtained descriptors and those obtained from the reference image.

$$
(f * g)_{i} \stackrel{\text { def }}{=} \sum_{j} f_{j}^{*} g_{i+j},
$$

where $i$ is dragging between the sequences relatively to each other and the superscript in the form of an asterisk means complex conjugation. If the number of similarities is more than some threshold value $N$, add the image to the series. If there are some images in the Copernicus system obtained within the decided time period, proceed to step 1 .

\section{Key point detectors and descriptors}

At the moment, there are many well-proven methods of identification (detection) of key points. The most widespread of them were investigated in the work.

One of them is the Harris method [6]. The principle of the method is that for the image under consideration $I$ a window $W$ is allocated with the center at the point $(x, y)$ then it is diddled towards $(u, v)$. The size of the selected window depends on the size of the image. Then the sum of squared differences between the initial and diddled window $(E(u, v))$, is calculated using the formula:

$$
\begin{aligned}
& E(u, v)=\sum_{(x, y) \in W} w(x, y)(I(x+u, y+v)-I(x, y))^{2} \approx, \\
& \approx \sum_{(x, y) \in W} w(x, y)\left(I_{x}(x, y) u+I_{y}(x, y) v\right)^{2} \approx(x, y) M\left(\begin{array}{l}
x \\
y
\end{array}\right)
\end{aligned}
$$

where $w(x, y)$ is impulsive response (Gaussian function is the most popular); $M$ is autocorrelation matrix:

$$
M=\sum_{(x, y) \in W} w(x, y)\left[\begin{array}{cc}
I_{x}^{2} & I_{x} I_{y} \\
I_{x} I_{y} & I_{y}^{2}
\end{array}\right],
$$

With large changes in the direction of the function $E(x, y)$ in the direction $x$ and $y$ large modulo the eigenvalues of the matrix $M$. are obtained. Due to the complexity the matrix eigenvalues calculations, a response measure $R$, is often used, determined from the formula: $R=\operatorname{det} M-k(\operatorname{tr} M)^{2}>k$, where $k$ is the empirical constant from the interval $[0,04 ; 0,06]$.

In this case the value $R$ will be positive for the angular key points. The local maxima of the response function in the neighbourhood of a given radius are calculated in the midst of the identified points, and these obtained points are selected as angular key points.

The advantage of the considered method is its stability to rotations and exceptionally to affine transformations of the image. But as for disadvantages, a significant sensitivity to noise in the image should be mentioned. There is one more detector of key point detection similar to the Harris method. It is the ShiTomasi angle detector [15], which differs in the calculation of the response measure. This method computes eigenvalues directly, so finding angles will be more stable. The bottom line lies in defining a threshold value and if the calculated value is more than the threshold, the point is considered an angle, in other words, a special point.

The above mentioned methods determine the key points working with the pixels of the image. There is an alternative approach, which involves the application of machine learning algorithms. For illustrative purpose of such methods the FAST method is chosen, the principle of which lies in building decision trees to classify pixels [13].

The method is based on the following: for each pixel $p$ we consider a circle with a radius of 4 pixels, inscribed in a square area with a side of 7 pixels. On the basis of the selected region, the importance of the point is concluded. Each of the pixels of the circle (the circle includes 16 pixels) relative to the pixel $\mathrm{p}$ can be in one of three States:

$$
S_{p \rightarrow x}=\left\{\begin{array}{lcl}
d, & I_{x} \leq I_{p}-t & \text { (darker) } \\
s, & I_{p}-t \leq I_{x} \leq I_{p}+t & \text { (similar). } \\
b, & I_{p}+t \leq I_{x} & \text { (lighter) }
\end{array}\right.
$$

For each $x$ and found $S_{p \rightarrow x}$ for each $p \in P$ ( $P$ is the variety of all pixels of the training image set) the set is divided into 3 subsets of points $P_{d}$ (darker), $P_{s}$ (similar), $P_{b}$ (lighter). Then the decision tree is built. According to the results of this decision tree, the angles on the test images are determined.

A key drawback of this approach connected with identifying special points is the order in which the points are selected and which influences the effectiveness of the work. It is also necessary to take into account the fact that there may be other key points in the environment of the initial point and in this case the method may be fallible.

Image distortion is a significant obstacle in the operation of detectors. This is because the algorithm may fail to detect key points on subsequent frames of the same area due to various frame changes. Images of the same area taken by different spacecraft may differ owing to the deviations in sensors, shooting conditions (position of the vehicle, season of the year, atmosphere). In the conducted researches the work of detectors was contrasted taking into consideration the following types of distortions: blurring and darkening (they can occur due to atmospheric phenomena). A number of images of the specified area were taken, then artificially with the help of graphic editors distortions such as darkening and blurring were made. After that, measurements of operating time took place and a number of points in the initial images was identified. Besides, the key points on the distorted images were also searched and the found points were compared with the reference ones. Thus, a percentage discrepancy between the key points of the reference image and the distorted one was defined.

Less resistant to blurring turned out to be the FAST method. With this type of distortion, the method makes an error of about $38 \%$ of the found points. For the same set of images, the Harries method made approximately $1.5 \%$ of errors. So did the ShiTomasi method. Thus, the invariance of the Harries and ShiTomasi methods in regard to distortions of blurring type can be confirmed. In the second type of distortions (darkening) the FAST method also gave worse results. In this case the error was about $43 \%$. And both methods: of Harries and of Shi-Tomasi showed invariance with respect to this type of distortion.

However, it is necessary to take into account the fact that these methods are adapted to the objects of artificial origin, while space images are more natural, that is they have got a more monotonous texture. Therefore, the criterion of the number of found key points is more important.

In studied images of the forest both Harries and Shi-Tomasi methods found approximately $73.16 \%$ fewer key points than the FAST method. At the same time the search time for points was approximately $78.57 \%$ of the search time consumed by the FAST method.

On the basis of the conducted researches it is possible to draw a conclusion about further application of the FAST method in the 
further experiments. Since the number of key points found in images is of higher priority than the invariance in respect to image distortions.

The second stage of the study was devoted to the application of detectors capable of determining the unsuitability of the image in the task of forest pathology monitoring based on the algorithm proposed in the previous section.

If the image is suitable, the FAST method finds about 89$93 \%$ of the reference points in the image. Fig. 1 shows pictures taken 23.05.2019 and 02.06.2019. Fig. 1 illustrates the results of the FAST method for these images with the coincidence of key points reaching $95 \%$.

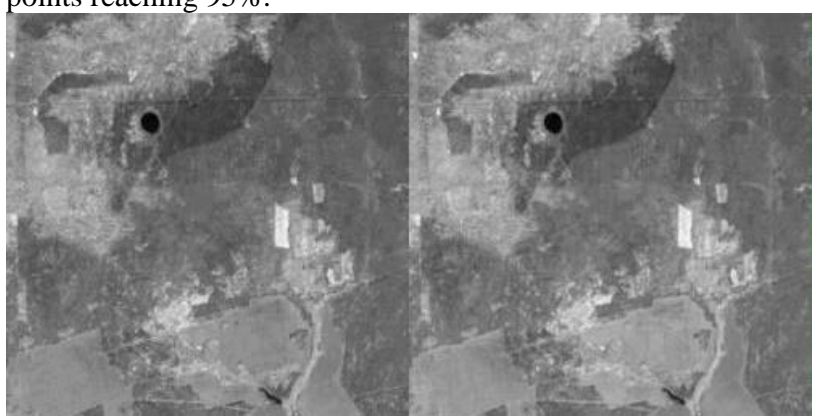

Fig 1. Satellite images of the lake «Krugloe» area, taken 23.05.2019and02.06.2019

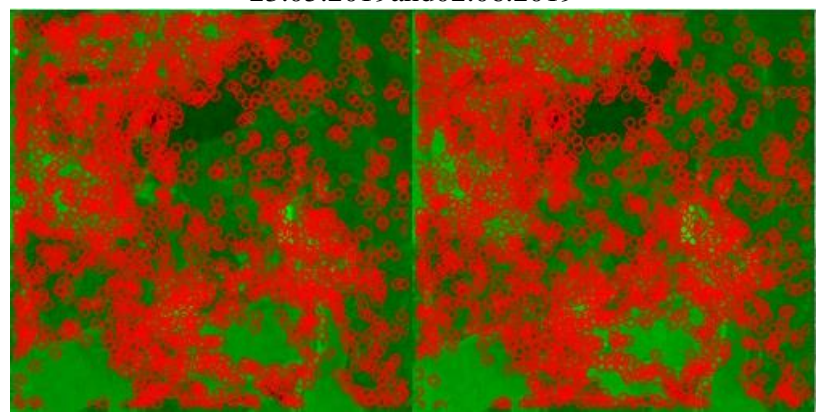

Fig 2. Key points of the FAST method for images in fig.1

However, in cases of the image impropriety, this method finds about $50-70 \%$ of the reference points. fig. 3 shows images taken 23.05.2019 and 25.06.2019 and fig. 4 shows the results of the FAST method, where the coincidence of key points is $70 \%$.

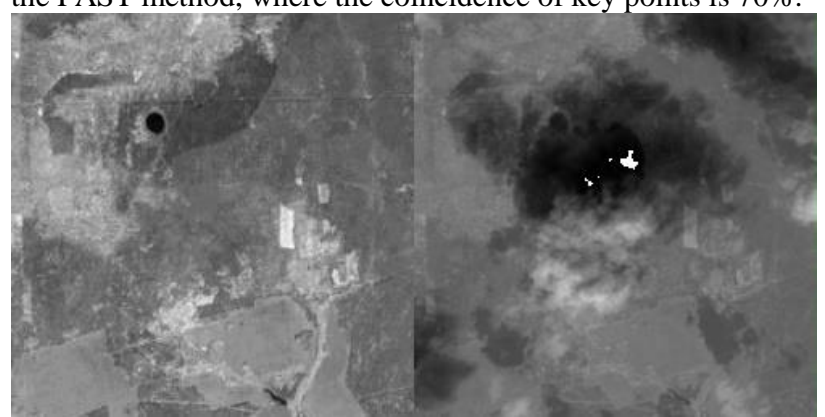

Fig. 3. Satellite images of the lake «Krugloe» area, taken 23.05.2019 и 25.05.2019

In addition to this investigation there were studies in regard to other methods, when the number of points is not as large as with the FAST method. These are Harries and Shi-Tomasi methods.

The results of similar test of Harries and Shi-Tomasi methods differ by about 2-3\%. In the case of good images, reference point correlation is approximately $80-95 \%$.

Exemplarily the results of the methods on the same images similar to the FAST method are presented (fig. 1). The Harries method matched $93 \%$ and the Shi-Tomasi method matched $95 \%$.

As applied to unsuitable images, the key point correlation is approximately $0-33 \%$. For purpose of illustration the results of the methods in the same images used by FAST method are presented (fig. 3). The Harries method as well as the Shi-Tomasi method had $0 \%$ of matching.

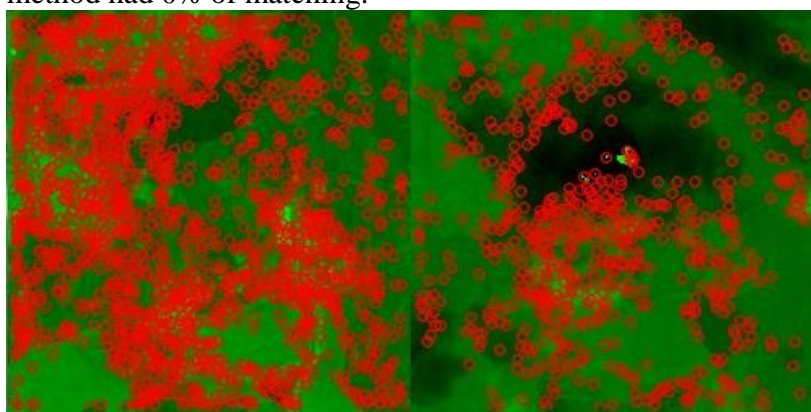

Fig. 4. Key points of the FAST method for images in fig. 3

All these conducted researches have resulted in the conclusion that Harries and Shi-Tomasi methods are more preferable than the FAST method. This is due to the fact that these two methods have a much larger difference between the thresholds of usable and unusable images comparing to the FAST method.

\section{Research results}

The final studies were the experiments aimed at finding satellite images free of clouds and other noise. To compare the results of the developed algorithm, the QGIS SAC module was used. The result of the search for images with $0 \%$ nebulosity in the SAC module accounted for 6 images, with a maximum nebulosity of $100 \%$ there were 61 images. The developed algorithm used in the same area and time limitations having the threshold value of suitable images equal to $89 \%$ gave the result of 9 images, which is $30 \%$ better than SAC.

To confirm the operation of the algorithm a manual selecting of all images took place, which resulted in 15 suitable images.

\section{Conclusion}

The article analyzes the process of forest pathology monitoring. The necessity of search automation applied for processing suitable forest images obtained from satellites was realized. Empirical studies connected with the application of key point detectors in the image were conducted in order to assess the applicability of the image for further processing and monitoring of forest pathology.

The studies revealed that a large number of key points is a hindrance in determining the suitability of the image, that is why the FAST detector was not introduced in further studies.

In the final experiments, it was confirmed that the proposed method of identifying suitable images for forest pathology monitoring produces results by $30 \%$ better than the well-proven QGIS SAC module. As a result, more images can be obtained for better tracking of pathology dynamics. However, it should be borne in mind that the result of the work depends not only on the chosen method of key point detection, but also upon the threshold value of the key point correlation accuracy

\section{References}

[1] Forest code of the Russian Federation as amended on December 27, 2018 (part 4, article 60.5)

[2] The order of April 5, $2017 \mathrm{~N} 156$ «On approval of the state forest pathology monitoring procedure».

[3] Showengerdt R. Remote sensing. Models and methods of image processing. M., 2010. $560 \mathrm{p}$.

[4] Coluzzi, Rosa \& Imbrenda, Vito \& Maria, Lanfredi \& Tiziana, Simoniello. (2018). A first assessment of the Sentinel-2 Level 1-C cloud mask product to support informed surface analyses. Remote Sensing of Environment. 217. 426-443. 10.1016/j.rse.2018.08.009. 
[5] Copernicus Open Access Hub. - URL: https://scihub.copernicus.eu.

[6] Harris, C and Stephens, M (1988). "A Combined Corner and Edge Detector". Alvey Vision Conference

[7] Hughes, M. J., Hayes, D. J. "Automated detection of cloud and cloud shadow in single-date Landsat imagery using neural networks and spatial post-processing", Remote Sens., vol. 6, no. 6, pp. 4907-4926, 2014.

[8] Jeppesen, J.H., Jacobsen, R.H., Inceoglu, F., Toftegaard, T.S. A cloud detection algorithm for satellite imagery based on deep learning. Remote Sensing of Environment, 229, 2019, pp. 247-259. https://doi.org/10.1016/j.rse.2019.03.039.

[9] Kwan, C., Hagen, L., Chou, B. et al. Simple and effective cloud- and shadow-detection algorithms for Landsat and Worldview images. SIViP, 2019, pp. 1-9. https://doi.org/10.1007/s11760-019-01532-2

[10] Man D.C., Luu V.H., Hoang V.T., Bui Q.H., Nguyen T.N.T. (2015) Cloud Detection Algorithm for LandSat 8 Image Using Multispectral Rules and Spatial Variability. In: Nguyen VH., Le AC., Huynh VN. (eds) Knowledge and Systems Engineering. Advances in Intelligent Systems and Computing, vol 326. Springer, Cham

[11] Pettorelli, N., Vik, J. O., Mysterud, A., Gaillard, J.-M., Tucker, C. J., Stenseth, N. C. Using the satellite-derived NDVI to assess ecological responses to environmental change // Trends in Ecology and Evolution. 2005. Vol. 20. P. 503-510. DOI: 10.1016/j.tree.2005.05.011.

[12] Qi, J., Chehbouni, A., Huete, A.R., Kerr, Y.H., Sorooshian, S. A modified soil adjusted vegetation index. Remote Sensing of Environment. V. 48, № 2, 1994, pp. 119-126.

[13] Rosten, Edward; Tom Drummond (2005). Fusing points and lines for high performance tracking. IEEE International Conference on Computer Vision. 2. pp. 1508-1511. CiteSeerX 10.1.1.60.4715. doi:10.1109/ICCV.2005.104. ISBN 978-0-7695-2334-7.

[14] Scaramuzza, P.L., Bouchard, M.A., Dwyer, J.L.: Development of the Landsat data continuity mission cloudcover assessment algorithms. IEEE Trans. Geosci. Remote Sens. 50(4), 1140-1154 (2012)

[15] Shi, J. and Tomasi, C. (June 1994). "Good Features to Track". 9th IEEE Conference on Computer Vision and Pattern Recognition. Springer. pp. 593-600. CiteSeerX 10.1.1.36.2669. doi:10.1109/CVPR.1994.323794

[16] Shiffman, S. Cloud detection from satellite imagery: a comparison of expert-generated and automatically generated decision trees. In Proceedings of the Eighth Workshop on Mining Scientific and Engineering Datasets, held in conjunction with the 2005 SIAM International Conference on Data Mining, April 21-23, Newport Beach, CA, 2005.

[17] Suzanne Angeli, Arnaud Quesney and Lydwine Gross (November 21st 2012). Image Simplification Using Kohonen Maps: Application to Satellite Data for Cloud Detection and Land Cover Mapping, Applications of SelfOrganizing Maps, Magnus Johnsson, IntechOpen, DOI: $10.5772 / 51352$.

[18] Tucker C. J. Red and photographic infrared linear combinations for monitoring vegetation // Rem. Sens. of Env. 1979. Vol. 8. P. 127-150. DOI: 10.1016/00344257(79)90013-0.

[19] Xie, F., Shi,M., Shi, Z., Yin, J., Zhao, D.: Multi-level Cloud Detection in Remote Sensing Images Based on Deep Learning. IEEE J. Sel. Top. Appl. Earth Obs. Remote Sens. 10(8), 3631-3640 (2017)

[20] Zhu, Z., et al., Improvement and expansion of the Fmask algorithm: cloud, cloud shadow, and snow detection for Landsats 4-7, 8, and Sentinel 2 images, Remote Sensing of
Environment

(2015), http://dx.doi.org/10.1016/j.rse.2014.12.014.

[21] Zi, Y., Xie, F., Jiang, Z.: A cloud detection method for Landsat 8 images based on PCANet. Remote Sens. 10(6), 877 (2018) 